\title{
EXISTENCE AND NONOSCILLATION THEOREMS FOR AN EMDEN-FOWLER EQUATION WITH DEVIATING ARGUMENT
}

\author{
BY
}

WILLIAM F. TRENCH

\begin{abstract}
Sufficient conditions are given for a generalized Emden-Fowler equation with deviating argument to have nonoscillatory solutions with prescribed asymptotic behavior as $t \rightarrow \infty$. The integrability condition on the nonlinear term requires only conditional convergence, supplemented by a condition on the order of convergence, which is automatically satisfied in some important special cases. The exponent in the nonlinear term may be any real number. The deviating argument is not assumed to be purely advanced or retarded, and, in some cases, need not tend to infinity. Some of the results are global, in that the desired solution is shown to exist on a given interval, rather than only for sufficiently large $t$.
\end{abstract}

1. Introduction. We consider the generalized Emden-Fowler functional equation

$$
\left(r(t) x^{\prime}(t)\right)^{\prime}+q(t) x(t)=p(t)|x(g(t))|^{\gamma} \operatorname{sgn} x(g(t))
$$

under the following standing assumption.

Assumption A. The function $g$ is continuously differentiable on $[a, \infty)$ and

$$
\min \left\{a, \inf _{t \geq a} g(t)\right\}=a_{0}>-\infty .
$$

The functions $r, p$, and $q$ are continuous and $r>0$ on $\left[a_{0}, \infty\right) ; p$ is not identically zero on any interval $[T, \infty)$. The equation

$$
\left(r(t) y^{\prime}\right)^{\prime}+q(t) y=0, \quad t>a_{0},
$$

has solutions $y_{1}$ and $y_{2}$ such that

$$
\begin{gathered}
y_{2}^{\prime} y_{1}-y_{2} y_{1}^{\prime}=1 / r, \\
y_{1}(t)>0, \quad y_{2}(t)>0, \quad t \geq a_{0},
\end{gathered}
$$

and

$$
\lim _{t \rightarrow \infty}\left(y_{2}(t) / y_{1}(t)\right)=\infty .
$$

Received by the editors February 21, 1985. Presented to the Society, January 11, 1986 at the 92nd Annual Meeting.

1980 Mathematics Subject Classification. Primary 34C11; Secondary 34K25.

Key words and phrases. Emden-Fowler nonoscillatory equation, asymptotic, global existence, local existence. 
It is well known $\left[3\right.$, p. 355] that if $(2)$ has a solution with no zeros on $\left[a_{0}, \infty\right)$, then it has solutions $y_{1}$ and $y_{2}$ which satisfy (3)-(5). For convenience below, we define $\rho=y_{2} / y_{1}$. From (3) and (5),

$$
\rho^{\prime}=1 / r y_{1}^{2} \quad \text { and } \quad \lim _{t \rightarrow \infty} \rho(t)=\infty \text {. }
$$

Throughout the paper all quantities are real, and we use " $O$ " and " $O$ " in the standard way to refer to behavior as $t \rightarrow \infty$. For each $b \geq a$, we define

$$
b_{0}=\min \left\{b, \inf _{t \geq b} g(t)\right\} .
$$

We say that $x$ is a solution of $(1)$ on $(b, \infty)$ if $x \in C\left[b_{0}, \infty\right), x^{\prime} \in C[b, \infty)$ and (1) holds for $t>b$.

We give sufficient conditions for (1) to have a solution $\hat{x}$ which, roughly speaking for now, exhibits one of the following general types of asymptotic behavior as $t \rightarrow$ $\infty$ :

$$
\hat{x}=c y_{i}+o\left(y_{1}\right) \quad \text { for } i=1 \text { or } 2,
$$

or

$$
\hat{x}=(c+o(1)) y_{2},
$$

where $c$ is a given nonzero constant. We believe that the results are unusual, for the following reasons:

(A) Some are global, in that the specified solution is shown to exist on a given interval, rather than only for $t$ sufficiently large. (For other global existence theorems for nonlinear equations, see Nehari [10], Noussair and Swanson [11] and Kusano and Trench $[\mathbf{7}, \mathbf{8}]$.)

(B) Specific estimates are given for the error $\hat{x}-c y_{i}$, and estimates are given for $\left(\hat{x} / y_{i}\right)^{\prime}$ in the case $(8)$, or $\left(\hat{x} / y_{2}\right)^{\prime}$ in $(9)$.

(C) The exponent $\gamma$ may be any real number. With few exceptions (e.g., Graef, Grammatikopoulos and Spikes [1], Kusano and Swanson [6], Taliaferro [12] and Trench $[\mathbf{1 4}, \mathbf{1 6}, \mathbf{1 7}])$, the extensive literature on the Emden-Fowler equation is confined to the case where $\gamma>0$.

(D) We place no restriction on the signs of $g^{\prime}(t)$ and $g(t)-t$, and, in some of our results, we do not require that $\lim _{t \rightarrow \infty} g(t)=\infty$.

(E) Our integrability conditions on $f$ permit conditional convergence of some of the improper integrals which occur. This continues a theme that we have developed in several recent papers (see, e.g., $[\mathbf{1 3 - 1 7}])$.

For other examples of asymptotic theory based on perhaps conditionally integrable perturbing terms, see the recent paper of Naito [9] on nonlinear second order equations. Examples dealing with linear equations are to be found in Hartman [3, pp. 375-384] and Hallam [2].

Kusano and Naito [4] and Kusano and Onose [5] have obtained results on oscillation of solutions of a class of functional equations which includes (1) with $q=0$.

It is convenient to collect certain technical definitions in the following standing assumption.

ASSUMPTION B. Let $c$ and $\theta$ be given constants, with $c \neq 0$ and $0<\theta<1$. Let $\gamma$ be an arbitrary real number, and define

$$
K_{1}=|\gamma|(1 \pm \theta)^{\gamma-1}, \quad K_{2}=|\gamma-1|(1 \pm \theta)^{\gamma-2} \theta+(1 \pm \theta)^{\gamma-1}
$$


where the " \pm " in each case is " + " if the exponent is nonnegative, or "-" if it is negative. For each $b \geq a$, let $b_{0}$ be as defined in (7), and define

$$
t(b)= \begin{cases}t & \text { if } t \geq b \\ b & \text { if } b_{0}<t<b\end{cases}
$$

Let $\phi$ be a function which is positive and nonincreasing on $[a, \infty)$, such that either $\lim _{t \rightarrow \infty} \phi(t)=0$ or $\phi=$ constant.

2. Existence of solutions which satisfy (8). In this section we need the following elementary extension of Abel's theorem on convergence of improper integrals. Its proof is based on (6) and integration by parts [17, Lemma 1].

Lemma 1. Suppose $P \in C[b, \infty)$ with $b \geq a$, and $\int^{\infty} y_{2}(s) P(s) d s$ converges (perhaps conditionally). Let

$$
\nu(t)=\sup _{\tau \geq t}\left|\int_{\tau}^{\infty} y_{2}(s) P(s) d s\right| .
$$

Then

$$
\left|\int_{t(b)}^{\infty}\left[y_{2}(s) y_{1}(t)-y_{1}(s) y_{2}(t)\right] P(s) d s\right| \leq \nu(t(b)) y_{1}(t), \quad t \geq b_{0}
$$

(see (7) and (11)), and

$$
\left|\int_{t}^{\infty} y_{1}(s) P(s) d s\right| \leq 2 \nu(t) / \rho(t), \quad t \geq b .
$$

THEOREM 1. Let $i=1$ or 2 . Suppose the integral

$$
F(t)=\int_{t}^{\infty} y_{2}(s) p(s)\left[y_{i}(g(s))\right]^{\gamma} d s
$$

converges (perhaps conditionally), and

$$
\sup _{r \geq t}|F(r)|=\psi(t) \leq \phi(t)
$$

Suppose also that the function

$$
R(t)=(\phi(t))^{-1} \int_{t}^{\infty}|F(s)| \phi(g(s)) \rho^{\prime}(g(s))[\rho(g(s))]^{-i}\left|g^{\prime}(s)\right| d s
$$

is defined and bounded on $(a, \infty)$, and that

$$
\sup _{\tau \geq b}\left\{\left(1+\theta K_{1}\right) \frac{\phi\left(b_{0}\right)}{\left[\rho\left(b_{0}\right)\right]^{i-1}} \frac{\psi(\tau)}{\phi(\tau)}+\theta|\gamma| K_{2} R(\tau)\right\}<\frac{\theta}{2}|c|^{-\gamma+1} .
$$

Then (1) has a solution $\hat{x}$ on $(b, \infty)$ such that

$$
\left|\frac{\hat{x}(t)}{y_{i}(t)}-c\right|<\theta|c| \frac{\left[\rho\left(b_{0}\right)\right]^{i-1}}{\phi\left(b_{0}\right)} \frac{\phi(t)}{[\rho(t)]^{i-1}}, \quad t \geq b_{0},
$$

and

$$
\left|\left(\frac{\hat{x}(t)}{y_{i}(t)}\right)^{\prime}\right| \leq \theta|c| \frac{\left[\rho\left(b_{0}\right)\right]^{i-1}}{\phi\left(b_{0}\right)} \frac{\phi(t) \rho^{\prime}(t)}{[\rho(t)]^{i}}, \quad t \geq b_{0}
$$


moreover, even if $\phi=$ constant,

$$
\frac{\hat{x}}{y_{i}}=c+o\left(\rho^{-i+1}\right)
$$

and

$$
\left(\frac{\hat{x}}{y_{i}}\right)^{\prime}=o\left(\rho^{-i} \rho^{\prime}\right)
$$

REMARK 1. The other assumptions of Theorem 1 imply that the left side of (15) is finite if $b \geq a$. We will obtain specific results from Theorem 1 by imposing various conditions which imply the inequality.

For purposes of motivation, we present the lengthy proof of this theorem informally. We assume throughout that $c>0$, which imposes no loss of generality.

We use the contraction mapping principle. It is easily verified that if $\hat{x}$ is continuous and positive on $\left[b_{0}, \infty\right)$ and satisfies the equation

$$
\hat{x}(t)=c y_{i}(t)+\int_{t(b)}^{\infty}\left[y_{2}(s) y_{1}(t)-y_{1}(s) y_{2}(t)\right] p(s)[\hat{x}(g(s))]^{\gamma} d s, \quad t \geq b_{0}
$$

(recall $(11)$ ), then $\hat{x}$ satisfies $(1)$ on $(b, \infty)$ and has the asymptotic behavior $(8)$. This suggests that we seek the desired solution $\hat{x}$ as a fixed point of the transformation F defined by

$$
(\mp x)(t)=c y_{i}(t)+\int_{t(b)}^{\infty}\left[y_{2}(s) y_{1}(t)-y_{1}(s) y_{2}(t)\right] p(s)[x(g(s))]^{\gamma} d s, \quad t \geq b_{0} .
$$

However, in order to obtain our results under milder assumptions, we wish to work with a transformation defined on a subset of $C^{\prime}\left[b_{0}, \infty\right)$, and, since our assumptions impose no conditions on $y_{1}^{\prime}$ and $y_{2}^{\prime}$, but only on $\left(y_{2} / y_{1}\right)^{\prime}=\rho^{\prime}$, we consider instead the transformation

$$
(\tau z)(t)=-\int_{t(b)}^{\infty}\left[1-\frac{\rho(t)}{\rho(s)}\right] F^{\prime}(s)[c+z(g(s))]^{\gamma} d s
$$

if $i=1$, or

$$
(\tau z)(t)=\int_{t(b)}^{\infty}\left[\frac{1}{\rho(s)}-\frac{1}{\rho(t)}\right] F^{\prime}(s)[c+z(g(s))]^{\gamma} d s
$$

if $i=2$. These choices were motivated by rewriting (20) in terms of the relative error

$$
\hat{z}=\left(\hat{x}-c y_{i}\right) / y_{i} \text {. }
$$

Thus, if $\tau \hat{z}=\hat{z}$, then solving (23) for $\hat{x}$ yields a solution of (1).

Formally differentiating (21) and (22) yields

$$
(\tau z)^{\prime}(t)=\rho^{\prime}(t) \int_{t(b)}^{\infty} \frac{F^{\prime}(s)}{\rho(s)}[c+z(g(s))]^{\gamma} d s
$$

if $i=1$, or

$$
(\tau z)^{\prime}(t)=\frac{\rho^{\prime}(t)}{\rho^{2}(t)} \int_{t(b)}^{\infty} F^{\prime}(s)[c+z(g(s))]^{\gamma} d s
$$

if $i=2$. 
From Lemma 1 with

$$
P(t)=p(t)\left[y_{i}(g(t))\right]^{\gamma}[c+z(g(t))]^{\gamma}
$$

and (21) and (24) (if $i=1$ ) or (22) and (25) (if $i=2$ ), it can be shown that if $z \in C\left[b_{0}, \infty\right)$ and the integral

$$
I(t ; z)=\int_{t}^{\infty} F^{\prime}(s)[c+z(g(s))]^{\gamma} d s
$$

exists and satisfies $I(t ; z)=O(\phi(t))$ on $[b, \infty)$, then $\tau z \in C^{\prime}\left[b_{0}, \infty\right)$ and

$$
\tau z=O\left(\phi \rho^{-i+1}\right), \quad(\tau z)^{\prime}=O\left(\phi \rho^{\prime} \rho^{-i}\right) .
$$

This motivates our decision that the domain of $\tau$ should be a suitable subset of the Banach space

$$
B=\left\{z \in C^{\prime}\left[b_{0}, \infty\right) \mid z=O\left(\phi \rho^{-i+1}\right), z^{\prime}=O\left(\phi \rho^{\prime} \rho^{-i}\right)\right\}
$$

with norm

$$
\|z\|=\sup _{t \geq b_{0}} \max \left\{\frac{|z| \rho^{i-1}}{\phi}, \frac{\left|z^{\prime}\right| \rho^{i}}{\phi \rho^{\prime}}\right\} .
$$

We will show that $\tau$ is a contraction on a certain sphere around the origin in $B$, provided that (15) holds.

The following lemma, which is valid for $i=1$ or 2 , will be useful below. We leave it to the reader to verify that it is merely a reformulation of Lemma 1.

LEMMA 2. Suppose that the assumptions of Theorem 1 hold. Let $h \in C[b, \infty)$ and suppose that the integral $\int^{\infty} F^{\prime}(s) h(s) d s$ converges (perhaps conditionally) and satisfies the inequality

$$
\sup _{r \geq t}(\phi(r))^{-1}\left|\int_{r}^{\infty} F^{\prime}(s) h(s) d s\right| \leq \lambda(t), \quad t \geq b
$$

where $\lambda$ is nonincreasing and continuous on $[b, \infty)$. Let

$$
J(t ; h)=-\int_{t(b)}^{\infty}\left[1-\frac{\rho(t)}{\rho(s)}\right] F^{\prime}(s) h(s) d s, \quad t \geq b_{0},
$$

if $i=1$, or

$$
J(t ; h)=\int_{t(b)}^{\infty}\left[\frac{1}{\rho(s)}-\frac{1}{\rho(t)}\right] F^{\prime}(s) h(s) d s, \quad t \geq b_{0}
$$

if $i=2$. Then $J(\cdot ; h) \in C^{\prime}\left[b_{0}, \infty\right)$, and

$$
|J(t ; h)| \leq \frac{\lambda(t(b)) \phi(t)}{[\rho(t)]^{i-1}}, \quad\left|J^{\prime}(t ; h)\right| \leq \frac{2 \lambda(t(b)) \phi(t) \rho^{\prime}(t)}{[\rho(t)]^{i}}
$$

hence, $J(\cdot ; h) \in B$ and $\|J(\cdot ; h)\| \leq 2 \lambda(b)$.

LEMMA 3. Let

$$
\hat{B}=\left\{z \in B \mid\|z\| \leq \theta c\left[\rho\left(b_{0}\right)\right]^{i-1} / \phi\left(b_{0}\right)\right\},
$$


where $c>0$ and $0<\theta<1$ (see (27) and (28)). Suppose that $z_{1}, z_{2} \in \hat{B}$ and $\tau \geq b_{0}$. Then

$$
\left|\left(c+z_{1}(\tau)\right)^{\gamma}-\left(c+z_{2}(\tau)\right)^{\gamma}\right| \leq K_{1} c^{\gamma-1}\left\|z_{1}-z_{2}\right\| \phi(\tau)(\rho(\tau))^{-i+1}
$$

and

$$
\begin{array}{r}
\left|\left(c+z_{1}(\tau)\right)^{\gamma-1} z_{1}^{\prime}(\tau)-\left(c+z_{2}(\tau)\right)^{\gamma-1} z_{2}^{\prime}(\tau)\right| \\
\leq K_{2} c^{\gamma-1}\left\|z_{1}-z_{2}\right\| \phi(\tau) \rho^{\prime}(\tau)(\rho(\tau))^{-i}
\end{array}
$$

where $K_{1}$ and $K_{2}$ are as defined in (10).

PROOF. If $z \in \hat{B}$ and $\tau \geq b_{0}$, then

$$
|z(\tau)| \leq\|z\| \phi(\tau)(\rho(\tau))^{-i+1} \leq\|z\| \phi\left(b_{0}\right)\left(\rho\left(b_{0}\right)\right)^{-i+1} \leq c \theta ;
$$

hence

$$
0<c(1-\theta) \leq c+z(\tau) \leq c(1+\theta),
$$

and, if $\alpha$ is an arbitrary real number, then

$$
(c+z(\tau))^{\alpha} \leq c^{\alpha}(1 \pm \theta)^{\alpha},
$$

with " \pm " chosen as specfied in Assumption B. The inequalities (31) and (32) can be obtained by applying the mean value theorem to the functions $g_{1}(u)=u^{\gamma}$ and $g_{2}(u, v)=u^{\gamma-1} v$, respectively, and invoking (33) and the definition of $\|z\|$. We omit the routine details.

We will use Lemma 2 to show that $\tau$ is a contraction of $\hat{B}$ into itself if (15) holds. To this end, suppose that $b \leq t \leq T$ and $z_{1}, z_{2} \in \hat{B}$, and consider the integral

$$
H_{T}\left(t ; z_{1}, z_{2}\right)=\int_{t}^{T} F^{\prime}(s)\left[\left[c+z_{1}(g(s))\right]^{\gamma}-\left[c+z_{2}(g(s))\right]^{\gamma}\right] d s .
$$

Integrating by parts yields

$$
\begin{aligned}
H_{T}\left(t ; z_{1}, z_{2}\right)= & \left.F(s)\left[\left[c+z_{1}(g(s))\right]^{\gamma}-\left[c+z_{2}(g(s))\right]^{\gamma}\right]\right|_{t} ^{T} \\
& -\gamma \int_{t}^{T} F(s)\left[\left[c+z_{1}(g(s))\right]^{\gamma-1} z_{1}^{\prime}(g(s))\right. \\
& \left.-\left[c+z_{2}(g(s))\right]^{\gamma-1} z_{2}^{\prime}(g(s))\right] g^{\prime}(s) d s .
\end{aligned}
$$

Since $\lim _{T \rightarrow \infty} F(T)=0$, the first member on the right side of (35) converges as $T \rightarrow \infty$. Moreover, (31) and (32) (with $\tau=g(s)$ ) and our integrability assumption in (14) imply that the integral in (35) converges absolutely. Therefore, the function $H\left(t ; z_{1}, z_{2}\right)=\lim _{T \rightarrow \infty} H_{T}\left(t ; z_{1}, z_{2}\right)$ exists for $t \geq b$. Moreover, routine estimates based on (13), (14), (31), (32) and (35) imply that

$$
\left|H\left(t ; z_{1}, z_{2}\right)\right| \leq c^{\gamma-1}\left\|z_{2}-z_{1}\right\| M(t) \phi(t),
$$

where

$$
M(t)=K_{1} \frac{\psi(t) \phi(g(t))}{\phi(t)[\rho(g(t))]^{i-1}}+|\gamma| K_{2} R(t) .
$$

Now we see that the integral $I(t ; z)$ in $(26)$ converges, since we can write it as

$$
I(t ; z)=-c^{\gamma} F(t)+H(t ; z, 0)
$$


(see (12) and (34)); moreover, from (13) and (36) (with $z_{1}=z, z_{2}=0$ ),

$$
|I(t ; z)| \leq c^{\gamma} \psi(t)+c^{\gamma-1}\|z\| M(t) \phi(t) .
$$

This and the restriction on $\|z\|$ for $z$ in $\hat{B}$ (see (30)) imply that

$$
|I(t ; z)| \leq c^{\gamma} N(t) \phi(t),
$$

where

$$
N(t)=\frac{\psi(t)}{\phi(t)}+\theta \frac{\left[\rho\left(b_{0}\right)\right]^{i-1}}{\phi\left(b_{0}\right)} M(t) .
$$

Because of (38) and Lemma 2 with $h(t)=[c+z(g(t))]^{\gamma}, \tau z \in B$ and

$$
\|\tau z\| \leq 2 c^{\gamma} \sup _{\tau \geq b} N(\tau) .
$$

Therefore, $\tau(\hat{B}) \subset \hat{B}$ if

$$
2 c^{\gamma-1} \sup _{\tau \geq b} N(\tau) \leq \theta\left[\rho\left(b_{0}\right)\right]^{i-1} / \phi\left(b_{0}\right)
$$

(see (30)). Because of (36) and Lemma 2 with

$$
h(t)=\left[c+z_{2}(g(t))\right]^{\gamma}-\left[c+z_{1}(g(t))\right]^{\gamma},
$$

it follows that

$$
\left\|\tau z_{2}-\tau z_{1}\right\| \leq 2 c^{\gamma-1}\left\|z_{2}-z_{1}\right\| \sup _{\tau \geq b} M(\tau),
$$

and therefore $T$ is a contraction if

$$
2 c^{\gamma-1} \sup _{\tau \geq b} M(\tau)<1 .
$$

However, by recalling (37) and (39), it can be seen that (15) implies both (40) and (41). Hence, $\tau$ is a contraction of $\hat{B}$ into itself, and therefore it has a unique fixed point $\hat{z}$; i.e., $\tau \hat{z}=\hat{z}$.

It is straightforward to verify that if $\hat{x}=(c+\hat{z}) y_{i}$ (see (23)), then $\hat{x}$ satisfies (1), (16) and (17). Since $\tau \hat{z}=\hat{z}$ and the integral in (21) or (22) converges if $z=\hat{z}$, (18) and (19) hold even if $\phi$ is constant. This completes the proof of Theorem 1.

Theorem 1 yields a global existence result if

$$
\gamma \neq 1,
$$

as follows.

THEOREM 2. Suppose the assumptions of Theorem 1 hold, except that (15) is replaced by (42). Let $b$ be any given number $\geq a$. Then (1) has a solution $\hat{x}$ on $[b, \infty)$ which satisfies (16)-(19), provided that $|c|$ is sufficiently small if $\gamma>1$, or sufficiently large if $\gamma<1$.

Proof. From Remark 1, (15) also holds if $|c|^{\gamma-1}$ is sufficiently large; hence, Theorem 1 implies the conclusion.

The following theorem provides more specific global results. 
THEOREM 3. Suppose the integral (12) converges, and let $\psi$ be as defined in (13). Suppose that $\gamma \neq 1$, and that $g^{\prime}$ is eventually sign-constant (either positive or negative). Define

$$
g(\infty)=\lim _{t \rightarrow \infty} g(t)
$$

Let any one of the following hypotheses hold:

$$
\begin{aligned}
& \left(\mathrm{H}_{1}\right) \quad g(\infty)<\infty \text {; } \\
& \left(\mathrm{H}_{2}\right) \quad g(\infty)=\infty \text { and } i=2 \text {; } \\
& \left(\mathrm{H}_{3}\right) \quad g(\infty)=\infty, i=1 \text { and } \\
& \int^{\infty} \frac{\rho^{\prime}(\tau) \psi(\tau)}{\rho(\tau)} d \tau<\infty \\
& \left(\mathrm{H}_{4}\right) \quad g(\infty)=\infty, i=1 \text {, } \\
& 0<A_{1} \leq \frac{\psi(g(t))}{\psi(t)} \leq A_{2}<\infty, \quad t \geq a
\end{aligned}
$$

and

$$
\int_{r}^{\infty} \frac{\rho^{\prime}(\tau) \psi^{2}(\tau)}{\rho(\tau)} d \tau=O(\psi(r)) .
$$

Then, if $b$ is a given number $\geq a,(1)$ has a solution $\hat{x}$ on $[b, \infty)$ such that

$$
\left|\frac{\hat{x}(t)}{y_{i}(t)}-c\right| \leq \theta|c| \frac{\left[\rho\left(b_{0}\right)\right]^{i-1} \psi(t)}{\psi\left(b_{0}\right)[\rho(t)]^{i-1}}, \quad t \geq b_{0},
$$

and

$$
\left|\left(\frac{\hat{x}(t)}{y_{i}(t)}\right)^{\prime}\right| \leq \theta|c| \frac{\left[\rho\left(b_{0}\right)\right]^{i-1}}{\psi\left(b_{0}\right)} \frac{\psi(t) \rho^{\prime}(t)}{[\rho(t)]^{i}}, \quad t \geq b_{0},
$$

provided that $|c|$ is sufficiently small if $\gamma>1$, or sufficiently large if $\gamma<1$.

ProOF. We apply Theorem 2 with $\psi=\phi$. We have only to verify that $R(t)$ is bounded. Since $|F(s)| \leq \psi(t)$ if $s \geq t$, the function $R$ in (14) satisfies the inequality

$$
R(t) \leq(\psi(t))^{-1} \int_{t}^{\infty} \psi(s) \psi(g(s)) \rho^{\prime}(g(s))[\rho(g(s))]^{-i}\left|g^{\prime}(s)\right| d s
$$

Invoking the eventual sign-constancy of $g^{\prime}$, the monotonicity of $\psi$ and the change of variable

$$
\tau=g(s)
$$

leads us from (49) to the inequality

$$
R(t) \leq\left|\int_{g(t)}^{g(\infty)} \psi(\tau) \rho^{\prime}(\tau)(\rho(\tau))^{-i} d \tau\right|
$$

valid for large $t$. Each of the hypotheses $\left(\mathrm{H}_{1}\right),\left(\mathrm{H}_{2}\right)$ and $\left(\mathrm{H}_{3}\right)$ implies that the integral in (51) converges (for $\left(\mathrm{H}_{2}\right)$, recall $(6)$ ), and that $R(t)=o(1)$. 
Now suppose $\left(\mathrm{H}_{4}\right)$ holds. From (45) and (49) (with $i=1$ ),

$$
R(t) \leq\left(A_{1} \psi(t)\right)^{-1} \int_{t}^{\infty} \psi^{2}(g(s)) \rho^{\prime}(g(s))[\rho(g(s))]^{-1} g^{\prime}(s) d s
$$

for large $t$. Now the change of variable (50) yields the inequality

$$
R(t) \leq\left(A_{1} \psi(t)\right)^{-1} \int_{g(t)}^{\infty} \psi^{2}(\tau)(\rho(\tau))^{-1} \rho^{\prime}(\tau) d \tau .
$$

Therefore, from (46),

$$
R(t) \leq K\left(A_{1} \psi(t)\right)^{-1} \psi(g(t))
$$

for some constant $K$, and (45) implies that $R(t)$ is bounded. This completes the proof.

REMARK 2. Notice that in the cases where $\left(\mathrm{H}_{1}\right)$ or $\left(\mathrm{H}_{2}\right)$ apply, the only integrability assumption in Theorem 3 is the requirement that (12) converge, perhaps conditionally. The hypotheses $\left(\mathrm{H}_{3}\right)$ and $\left(\mathrm{H}_{4}\right)$ impose some demands on the order of convergence in (12), but they are mild in that they permit $\psi$ to decay quite slowly by comparison with $1 / \rho$ as $t \rightarrow \infty$. For example, if $\psi \sim K(\log )^{-\alpha}$, then (44) holds if $\alpha>2$, while (46) holds if $\alpha \geq 1$. Obviously, (47) holds in the important special case where $g(t)=t$, but this is by no means the only case for which this is true.

Theorem 1 also implies the following local (near infinity) theorem, which states that (1) has solutions with prescribed asymptotic behavior, defined for $t$ sufficiently large.

THEOREM 4. Suppose the integral (12) converges, and let $\psi$ be as in (13). Suppose also that

$$
\lim _{t \rightarrow \infty} g(t)=\infty
$$

and that

$$
\begin{gathered}
\varlimsup_{t \rightarrow \infty}(\psi(t))^{-1} \int_{t}^{\infty}|F(s)| \psi(g(s)) \rho^{\prime}(g(s))[\rho(g(s))]^{-i}\left|g^{\prime}(s)\right| d s \\
<|c|^{-\gamma+1} /\left(2|\gamma| K_{2}\right) .
\end{gathered}
$$

Then (1) has a solution $\hat{x}$ which satisfies (47) and (48) on $[b, \infty)$ provided that $b$ is sufficiently large.

Proof. With $\phi=\psi$, the function on the left of $(53)$ is $R(t)$ (see (14)). From (52), (53), and the definition (7) of $b_{0}$, there are numbers $\xi<1$ and $b$ sufficiently large such that

$$
R(\tau) \leq \xi|c|^{-\gamma+1} / 2|\gamma| K_{2}, \quad \tau \geq b
$$

and

$$
\left(1+\theta K_{1}\right) \psi\left(b_{0}\right)\left(\rho\left(b_{0}\right)\right)^{-i+1}<(1-\xi) \theta|c|^{-\gamma+1} / 2 .
$$

This implies (15) with $\phi=\psi$, and Theorem 1 implies the conclusion.

The following theorem applies in cases where

$$
\varliminf_{t \rightarrow \infty} g(t)<\infty,
$$

although it should be noted that this is not included in the hypotheses of the theorem. 
THEOREM 5. Suppose that the assumptions of Theorem 1 hold, except for (15). Suppose also that

$$
\lim _{t \rightarrow \infty} \psi(t) / \phi(t)=0
$$

and

$$
\varlimsup_{t \rightarrow \infty} R(t)<|c|^{-\gamma+1} / 2|\gamma| K_{2} .
$$

Then (1) has a solution $\hat{x}$ which satisfies (16) and (17) on $[b, \infty)$, provided that $b$ is sufficiently large.

PROOF. From (55) and (56), there are numbers $\xi<\frac{1}{2}$ and $b$ sufficiently large such that (54) holds and

$$
\left(1+\theta K_{1}\right) \frac{\phi\left(a_{0}\right)}{\left(\rho\left(a_{0}\right)\right)^{i-1}} \frac{\phi(\tau)}{\psi(\tau)}<\frac{(1-2 \xi) \theta|c|^{-\gamma+1}}{2}, \quad \tau \geq b .
$$

Since

$$
\phi\left(b_{0}\right)\left(\rho\left(b_{0}\right)\right)^{-i+1} \leq \phi\left(a_{0}\right)\left(\rho\left(a_{0}\right)\right)^{-i+1},
$$

(57) and (58) imply (15). Hence, Theorem 1 implies the conclusion.

COROLlaRY 1. Suppose the integral (12) converges and

$$
\int^{\infty}|F(s)| \rho^{\prime}(g(s)) \rho(g(s))^{-i}\left|g^{\prime}(s)\right| d s<\infty .
$$

Then (1) has a solution $\hat{x}$ on $[b, \infty)$ which satisfies (16) and (17) with $\phi=$ constant, provided that $b$ is sufficiently large. Moreover, $\hat{x}$ also satisfies (18) and (19).

3. Existence of solutions which satisfy (9). In this section we use some of the symbols used in $\S 2$, but with different meanings. This should not cause confusion, and it enables us to present proofs here more efficiently; however, it is important to realize that when we refer here to equations in $\S 2$, the symbols in the latter are to have the meanings assigned to them in this section.

THEOREM 6. Define

$$
\hat{\phi}(t)=(\rho(t))^{-1}\left|\int_{a}^{t} \rho^{\prime}(\tau) \phi(\tau) d \tau\right|, \quad t \geq a_{0}
$$

and

$$
\phi_{m}(t)=\sup _{r \geq t} \max \{\phi(r), \hat{\phi}(r)\}
$$

Suppose that the integral

$$
F(t)=\int_{t}^{\infty} y_{1}(s) p(s)\left[y_{2}(g(s))\right]^{\gamma} d s
$$

converges and satisfies (13), and that the function

$$
R(t)=(\phi(t))^{-1} \int_{t}^{\infty}|F(s)| \phi_{m}(g(s)) \rho^{\prime}(g(s))[\rho(g(s))]^{-1}\left|g^{\prime}(s)\right| d s
$$

is bounded on $[a, \infty)$. Now suppose that

$$
\sup _{\tau \geq b}\left\{\left(1+\theta K_{1}\right) \phi_{m}(b) \frac{\psi(\tau)}{\phi(\tau)}+\theta|\gamma| K_{2} R(\tau)\right\}<\frac{\theta|c|^{-\gamma+1}}{2} .
$$


Then (1) has a solution $\hat{x}$ on $(b, \infty)$ such that

$$
\left|\frac{\hat{x}(t)}{y_{2}(t)}-c\right| \leq \theta|c| \frac{\phi_{m}(t)}{\phi_{m}(b)}, \quad t \geq b,
$$

and

$$
\left|\left(\frac{\hat{x}(t)}{y_{2}(t)}\right)^{\prime}\right| \leq \theta|c| \frac{\phi_{m}(t)}{\phi_{m}(b)} \frac{\rho^{\prime}(t)}{\rho(t)}, \quad t \geq b
$$

moreover,

$$
\frac{\hat{x}}{y_{2}}=c+o(1)
$$

and

$$
\left(\frac{\hat{x}}{y_{2}}\right)^{\prime}=o\left(\frac{\rho^{\prime}}{\rho}\right)
$$

even if $\phi$ is constant.

REMARK 3. A comment is in order regarding the relative sizes of $\phi$ and $\phi_{m}$ as $t \rightarrow \infty$. Roughly speaking, $\phi$ and $\phi_{m}$ are of the same order of magnitude if $\phi$ does not approach zero too rapidly by comparison with $1 / \rho$ as $t \rightarrow \infty$, while

$$
\lim _{t \rightarrow \infty} \frac{\phi_{m}(t)}{\phi(t)}=\infty
$$

if it does. For example, if $\phi=K \rho^{-\alpha}$ with $0 \leq \alpha<1$, then

$$
\lim _{t \rightarrow \infty} \frac{\phi_{m}(t)}{\phi(t)}=\frac{1}{1-\alpha} \text {. }
$$

On the other hand, if $\phi=K \rho^{-\alpha}$ with $\alpha>1$, then

$$
\phi_{m}(t) \sim \frac{K(\rho(a))^{-\alpha+1}}{(\alpha-1) \rho(t)},
$$

so that

$$
\lim _{t \rightarrow \infty} \frac{\phi_{m}(t)}{\phi(t)}=\infty
$$

However, we would not use Theorem 6 in this case, since if the integral in (59) converges and

$$
\sup _{\tau \geq t}\left|\int_{\tau}^{\infty} y_{1}(s) p(s)\left[y_{2}(g(s))\right]^{\gamma} d s\right| \leq K(\rho(t))^{-\alpha}
$$

with $\alpha>1$, then integration by parts shows that the integral in (12) (with $i=2$ ) also converges, and that

$$
\sup _{\tau \geq t}\left|\int_{\tau}^{\infty} y_{2}(s) p(s)\left[y_{2}(g(s))\right]^{\gamma} d s\right| \leq \frac{K \alpha}{(\alpha-1)}(\rho(t))^{-\alpha+1} ;
$$

therefore, we would try to apply Theorem 1 with $i=2$ rather than Theorem 6 . It should be noted, however, that there are applications of Theorem 6 in which (66) 
occurs: for example, if $\phi \sim K / \rho$, then (12) may fail to converge with $i=2$, and $\phi_{m} \sim(K \log \rho) / \rho$.

Proof OF TheOREM 6. Again we assume that $c>0$. From (2) and (6), it is easily verified that if $\hat{x}$ is continuous and positive on $\left[b_{0}, \infty\right)$ and satisfies the equation

$$
\hat{x}(t)=c y_{2}(t)-y_{1}(t) \int_{b}^{t} \rho^{\prime}(\tau)\left(\int_{\tau}^{\infty} y_{1}(s) p(s)[\hat{x}(g(s))]^{\gamma} d s\right) d \tau,
$$

then $\hat{x}$ satisfies $(1)$ on $(b, \infty)$, and has the asymptotic behavior (9). This suggests that we seek the desired solution $\hat{x}$ as a fixed point of the transformation

$$
(\mathcal{F} x)(t)=\left\{\begin{array}{l}
c y_{2}(t), \quad b_{0} \leq t \leq b, \\
c y_{2}(t)-y_{1}(t) \int_{b}^{t} \rho^{\prime}(\tau)\left(\int_{\tau}^{\infty} y_{1}(s) p(s)[x(g(s))]^{\gamma} d s\right) d \tau, \quad t>b .
\end{array}\right.
$$

However, it is convenient to look instead for the relative error $\hat{z}=\left(\hat{x}-c y_{2}\right) / y_{2}$. Rewriting (67) in terms of $\hat{z}$ leads us to consider the transformation $\tau$ defined by

$$
(\tau z)(t)=\left\{\begin{array}{l}
0, \quad b_{0} \leq t \leq b, \\
(\rho(t))^{-1} \int_{b}^{t} \rho^{\prime}(\tau)\left(\int_{\tau}^{\infty} F^{\prime}(s)[c+z(g(s))]^{\gamma} d s\right) d \tau, \quad t>b,
\end{array}\right.
$$

with $F$ as in (59). We will apply $\tau$ to a certain sphere around the origin in the Banach space $B$ of functions $z$ such that

$$
z \in C\left[b_{0}, \infty\right) ; \quad z(t)=0, \quad b_{0} \leq t \leq b ; \quad z=O\left(\phi_{m}\right)
$$

and

$$
z^{\prime} \in C[b, \infty) ; \quad z^{\prime}=O\left(\phi_{m} \rho^{\prime} / \rho\right)
$$

with norm

$$
\|z\|=\sup _{t \geq b} \max \left\{\frac{|z|}{\phi_{m}}, \frac{\left|z^{\prime}\right| \rho}{\phi_{m} \rho^{\prime}}\right\} .
$$

The following lemma indicates why we chose this space. Its proof is straightforward.

LEMMA 4. Suppose that $h \in C[b, \infty)$ and the integral $\int^{\infty} F^{\prime}(s) h(s) d s$ converges (perhaps conditionally) and satisfies (29) with $\lambda$ nonincreasing and continuous on $[b, \infty)$. Let

$$
J(t ; h)=\left\{\begin{array}{l}
0, \quad b_{0} \leq t \leq b \\
(\rho(t))^{-1} \int_{b}^{t} \rho^{\prime}(\tau)\left(\int_{\tau}^{\infty} F^{\prime}(s) h(s) d s\right) d \tau, \quad t>b
\end{array}\right.
$$

Then

$$
|J(t ; h)| \leq(\rho(t))^{-1} \int_{b}^{t} \rho^{\prime}(\tau) \lambda(\tau) \phi(\tau) d \tau \leq \lambda(b) \hat{\phi}(t)
$$

and

$$
\begin{aligned}
\left|J^{\prime}(t ; h)\right| & \leq \rho^{\prime}(t)(\rho(t))^{-1}\left[\int_{b}^{t} \rho^{\prime}(\tau) \lambda(\tau) \phi(\tau) d \tau+\lambda(t) \phi(t)\right] \\
& \leq \rho^{\prime}(t)(\rho(t))^{-1}[\lambda(b) \hat{\phi}(t)+\lambda(t) \phi(t)] \\
& \leq 2 \lambda(b) \phi_{m}(t) \rho^{\prime}(t) / \rho(t) .
\end{aligned}
$$


Therefore, $J(\cdot ; h) \in B$ (as defined in (68) and (69)), with norm as defined in (70) satisfying the inequality $\|J(\cdot ; h)\| \leq 2 \lambda(b)$.

We now use Lemma 4 to show that $\tau$ is a contraction of the sphere

$$
\hat{B}=\left\{z \in B \mid\|z\| \leq \theta c / \phi_{m}(b)\right\}
$$

into itself if $(61)$ holds. We proceed as in the proof of Theorem 1 with $i=1$ (specifically, beginning with the sentence "To this end..." preceding (34), with $F$ as in (59)). This leads to the existence of $H\left(t ; z_{1}, z_{2}\right)$ for any $z_{1}, z_{2} \in \hat{B}$, and the estimate (36), except that $M(t)$ is slightly modified:

$$
M(t)=K_{1} \frac{\psi(t)}{\phi(t)} \phi_{m}(g(t))+|\gamma| K_{2} R(t) .
$$

(In applying Lemma 3 , we take $i=1$ and replace $\phi$ by $\phi_{m}$ and $b_{0}$ by $b$.)

The integral $I(t ; z)$ in (26) (with $F(t)$ as in (59) and $\hat{B}$ as in this proof) can again be shown to exist and satisfy (38), with (39) replaced by

$$
N(t)=\frac{\psi(t)}{\phi(t)}+\frac{\theta}{\phi_{m}(b)} M(t)
$$

whenever $z \in \hat{B}$. Applying Lemma 4 as we applied Lemma 2 in the proof of Theorem 1 now shows that $T$ is a contraction of $\hat{B}$ into itself if

$$
2 c^{\gamma-1} \sup _{\tau \geq b} N(\tau) \leq \theta / \phi_{m}(b) \text { and } 2 c^{\gamma-1} \sup _{\tau \geq b} M(\tau)<1,
$$

with $M(t)$ and $N(t)$ as defined in (71) and (72). Since (61) implies (73), the rest of the proof is like that of Theorem 1 .

Theorem 6 implies a global result analogous to Theorem 2, which we leave to the reader.

With $\psi(t)=\sup _{\tau \geq t}|F(\tau)|$, let

$$
\psi_{m}(t)=\sup _{r \geq t} \max \left\{\psi(r),(\rho(r))^{-1}\left|\int_{a}^{r} \rho^{\prime}(\tau) \psi(\tau) d \tau\right|\right\} .
$$

The proofs of the following theorems are similar to those of Theorems 3-5. We omit them.

THEOREM 7. Suppose that the integral (59) converges, that $\gamma \neq 1$, and that $g^{\prime}$ is eventually sign-constant. Let any one of the following hypotheses hold:

$\left(\tilde{\mathrm{H}}_{1}\right) g(\infty)<\infty($ see $(43))$.

$\left(\tilde{\mathrm{H}}_{3}\right) g(\infty)=\infty$ and

$$
\int^{\infty} \frac{\rho^{\prime}(\tau) \psi_{m}(\tau)}{\rho(\tau)} d \tau<\infty
$$

$\left(\tilde{\mathrm{H}}_{4}\right) g(\infty)=\infty$

$$
0<A_{1} \leq \frac{\psi(t)}{\psi_{m}(g(t))} \leq A_{2}<\infty, \quad t \geq a,
$$

and

$$
\int_{r}^{\infty} \frac{\psi_{m}^{2}(\tau) \rho^{\prime}(\tau)}{\rho(\tau)} d \tau=O(\psi(r))
$$


Then, if $b$ is a given number $\geq a,(1)$ has a solution $\hat{x}$ on $(b, \infty)$ such that

$$
\left|\frac{\hat{x}(t)}{y_{2}(t)}-c\right| \leq \theta|c| \frac{\psi_{m}(t)}{\psi_{m}(b)}, \quad t \geq b
$$

and

$$
\left|\left(\frac{\hat{x}(t)}{y_{2}(t)}\right)^{\prime}\right| \leq \theta|c| \frac{\psi_{m}(t) \rho^{\prime}(t)}{\psi_{m}(b) \rho(t)}, \quad t \geq b,
$$

provided that $|c|$ is sufficiently small if $\gamma>1$, or sufficiently large if $\gamma<1$.

THEOREM 8. Suppose that the integral (59) converges, and let $\psi$ be as in (13). Suppose also that $\lim _{t \rightarrow \infty} g(t)=\infty$ and

$$
\begin{gathered}
\varlimsup_{t \rightarrow \infty}(\psi(t))^{-1} \int_{t}^{\infty}|F(s)| \psi_{m}(g(s)) \rho^{\prime}(g(s))[\rho(g(s))]^{-1}\left|g^{\prime}(s)\right| d s \\
<|c|^{-\gamma+1} /\left(2|\gamma| K_{2}\right) .
\end{gathered}
$$

Then (1) has a solution $\hat{x}$ which satisfies (74) and (75) on $[b, \infty)$, provided that $b$ is sufficiently large.

THEOREM 9. Suppose that the assumptions of Theorem 6 hold, except for (61). Suppose also that (55) and (56) hold, with $R(t)$ as defined in (60). Then (1) has a solution $\hat{x}$ which satisfies (62) and (63) on $[b, \infty)$, provided that $b$ is sufficiently large.

COROLlARY 2. Suppose that the integral (59) converges and

$$
\int^{\infty}|F(s)| \rho^{\prime}(g(s))[\rho(g(s))]^{-1}\left|g^{\prime}(s)\right| d s<\infty .
$$

Then (1) has a solution $\hat{x}$ which satisfies (62) and (63) with $\phi_{m}=$ constant on $[b, \infty)$, provided that $b$ is sufficiently large. Moreover, $\hat{x}$ also satisfies $(64)$ and $(65)$.

\section{REFERENCES}

1. J. R. Graef, M. K. Grammatikopoulos and P. W. Spikes, On the positive solutions of a higher order differential equation with a discontinuity, Internat. J. Math. Math. Sci. 5 (1982), 263-273.

2. T. G. Hallam, Asymptotic integration of second order differential equations with integrable coefficients, SIAM J. Appl. Math. 19 (1970), 430-439.

3. P. Hartman, Ordinary differential equations, Wiley, New York, 1964.

4. T. Kusano and M. Naito, Nonlinear oscillation of second order differential equations with retarded argument, Ann. Mat. Pura Appl. 106 (1976), 171-185.

5. T. Kusano and H. Onose, Nonlinear oscillation of second order functional differential equations with advanced arguments, J. Math. Soc. Japan 29 (1977), 541-559.

6. T. Kusano and C. A. Swanson, Asymptotic properties of semilinear elliptic equations, Funkcial. Ekvac. 26 (1983), 115-129.

7. T. Kusano and W. F. Trench, Global existence theorems for solutions of nonlinear differential equations with prescribed asymptotic behavior, J. London Math. Soc. 31 (1985), 478-486.

8. _ Existence of global solutions with prescribed asymptotic behavior for nonlinear ordinary differential equations, Ann. Mat. Pura Appl. (to appear).

9. M. Naito, Asymptotic behavior of solutions of second order equations with integrable coefficients, Trans. Amer. Math. Soc. 282 (1984), 577-588. 
10. Z. Nehari, On a class of nonlinear second-order differential equations, Trans. Amer. Math. Soc. 95 (1960), 101-123.

11. E. S. Noussair and C. A. Swanson, Global positive solutions of semilinear elliptic equations, Canad. J. Math. 35 (1983), 839-866.

12. S. Taliaferro, On the positive solutions of $y^{\prime \prime}+\phi(t) y^{-\lambda}=0$, Nonlinear Anal. 2 (1978), 437-446.

13. W. F. Trench, Asymptotic integration of linear differential equations subject to integral smallness conditions involving ordinary convergence, SIAM J. Math. Anal. 7 (1976), 213221.

14. __ Asymptotic integration of $y^{(n)}+P(t) y^{\gamma}=f(t)$ under mild integral conditions, Funkcial. Ekvac. 26 (1983), 197-209.

15. __ Asymptotic integration of linear differential equations subject to mild integral conditions, SIAM J. Math. Anal. 5 (1984), 932-942.

16. _ Asymptotic behavior of solutions of an nth order differential equation, Rocky Mountain J. 14 (1984), 441-450.

17. _ Functional perturbations of second order differential equations, SIAM J. Math. Anal. 16 (1985), 741-756.

Department of Mathematics and Computer Science, Drexel University, Philadelphia, Pennsylvania 19104 